\title{
Endoscopic Ultrasonography-Guided Gastroenterostomy Techniques for Treatment of Malignant Gastric Outlet Obstruction
}

\author{
Ryosuke Tonozuka', Takayoshi Tsuchiya', Shuntaro Mukai', Yuichi Nagakawa ${ }^{2}$ and Takao Itoi' \\ Department of ${ }^{1}$ Gastroenterology and Hepatology, ${ }^{2}$ Gastrointestinal and Pediatric Surgery, Tokyo Medical University, Tokyo, Japan
}

Gastric outlet obstruction (GOO) can be caused by periampullary malignancies and often leads to a reduction in a patient's quality of life. Recently, endoscopic ultrasonography-guided gastroenterostomy (EUS-GE) using a lumen-apposing self-expandable metal stent (LAMS) has been developed as a minimally invasive and durable endoscopic treatment for GOO. There are three types of EUS-GE technique: (1) the direct technique; (2) device-assisted techniques, such as a balloon catheter, nasobiliary drainage tube, and ultraslim endoscopy; and (3) EUS-guided double balloon-occluded gastrojejunostomy bypass. Previous reports of EUS-GE with LAMS have shown technical and clinical success rates (regardless of technique and etiology) of $87 \%-100 \%$ and $84 \%-100 \%$, respectively. Studies comparing EUS-GE and surgical gastrojejunostomy have shown similar success rates, reintervention rates, and cost benefits, with a lower rate of early adverse events in EUS-GE. A comparison of EUS-GE and endoscopic enteral stent placement revealed similar technical success rates, but initial clinical success rate was higher and the rate of stent failure requiring reintervention was lower with EUS-GE. Clin Endosc 2020;53:510-518

Key Words: Duodenal obstruction; Endosonography; Gastric bypass; Gastric outlet obstruction; Gastroenterostomy

\section{INTRODUCTION}

Gastric outlet obstruction (GOO) frequently occurs in patients with periampullary malignancies (i.e., cancers originating from the head of the pancreas, the distal bile duct, duodenum, or ampulla) or gastric cancer, with symptoms such as abdominal pain, postprandial vomiting, and inability to tolerate oral intake, which lead to a reduction in a patient's quality of life (QOL). ${ }^{1}$ Therefore, relieving GOO symptoms is crucial to maintain an adequate nutritional status and improve

Received: June 8, 2020 Revised: July 19, 2020

Accepted: July 21, 2020

Correspondence: Ryosuke Tonozuka

Department of Gastroenterology and Hepatology, Tokyo Medical University, 6-71 Nishishinjuku, Shinjuku-ku, Tokyo 160-0023, Japan

Tel: +81-3-3342-6111, Fax: +81-3-5381-6654, E-mail: tonozuka1978@gmail.com ORCID: https://orcid.org/0000-0003-3999-2847

(cc) This is an Open Access article distributed under the terms of the Creative Commons Attribution Non-Commercial License (http://creativecommons.org/ licenses/by-nc/3.0) which permits unrestricted non-commercial use, distribution, and reproduction in any medium, provided the original work is properly cited. a patient's QOL.,3 Considering the systemic condition of these patients and their limited prognosis, minimally invasive treatments are preferred. Traditionally, either open or laparoscopic surgical gastrojejunostomy (SGJ) has been performed as the primary treatment for malignant GOO, and has resulted in a high rate of complications. More recently, the endoscopic enteral self-expandable metal stent placement (EES) technique was developed, with the aim to decrease the invasiveness of SGJ. ${ }^{4}$ Compared to SGJ, EES is associated with more favorable short-term outcomes, including lower complication rates, shorter time to restart oral feeding, and shorter hospital stay, but has a higher rate of stent obstruction, and requires repeated treatment. ${ }^{5,6}$ Therefore, SGJ may be more suitable than EES for patients with a relatively long expected survival. ${ }^{7}$ In recent years, endoscopic ultrasonography-guided gastroenterostomy (EUS-GE) using a lumen-apposing self-expandable metal stent (LAMS) has been developed for the management of GOO, and various techniques have been developed for further improvement of its safety and efficacy. ${ }^{8-21}$ In this review, we describe the current status and perspectives of EUS-GE. 


\section{TERMINOLOGY AND INDICATIONS}

\section{Endoscopic ultrasonography-guided gastroenterostomy}

EUS-GE is an interventional EUS technique, in which an anastomosis of the stomach and enteric wall is created. EUSGE creates a shortcut in the food pathway, similar to a surgical bypass, which is expected to lead to a longer stent patency than conventional EES, owing to its shorter stent length and the fact that it is fully covered; besides, EUS-GE is theoretically thought to be less invasive than SGJ. ${ }^{7}$ EUS-GE procedures can be performed in two ways, one is EUS-gastroduodenostomy, by the puncturing the third or fourth portion of the duodenum, and the other is gastrojejunostomy, which targets the jejunum. Regardless of the etiology and size of the stenosis, symptomatic GOO is considered to be a candidate for EUSGE (Table 1). The small intestinal tract intended for stent deployment should be adjacent to the stomach. A preprocedural computed tomography scan in both transverse and coronal view is helpful in deciding on the puncture site as a preoperative roadmap. In patients with cancer invasion into the gastric wall, sufficient attention should be paid to find a safe puncture site. Furthermore, EUS-GE may be unsafe and technically difficult in cases of cancer extending into the fourth portion of the duodenum or the proximal jejunum around the ligament of Treitz. An absolute contraindication to performing EUS-GE is the presence of a large amount of ascites, which interferes with the adherence and fixation of the bowel loops. ${ }^{7}$ Patients with GOO symptoms often have a large amount of gastric food residue, which may disturb successful EUS-GE and lead to serious adverse events (AEs). Thus, patients should have no food intake, or a low-residue diet for several days until the scheduled EUS-GE. In addition, in cases with large volumes of gastric residual before performing the EUS-GE, it is desirable

Table 1. Diseases Indicated for Endoscopic Ultrasonography-Guided Gastroenterostomy

\begin{tabular}{ll}
\hline Malignant diseases & Benign diseases \\
\hline Gastric cancer & Gastric ulcer \\
\hline Duodenal cancer & Duodenal ulcer \\
\hline Pancreatic cancer & Acute pancreatitis \\
\hline Bile duct cancer & Chronic pancreatitis \\
\hline Gallbladder cancer & Postendoscopic therapy \\
\hline Ampullary cancer & Surgical anastomosis stricture \\
Lymph node metastasis & Superior mesenteric syndrome \\
Miscellaneous & Miscellaneous \\
\hline
\end{tabular}

to remove the residue using retrieval devices (e.g., forceps, snare, basket, or net catheter) in advance.

\section{Lumen-apposing self-expandable metal stent}

LAMS helps attach two adjacent organs and its usefulness in EUS-GE was demonstrated in a pig model of GOO. ${ }^{22}$ The stent is made of a shape memory alloy and is fully covered with silicone membrane to prevent fluid leakage. Standard LAMS includes the AXIOS ${ }^{\mathrm{TM}}$ stent (Boston Scientific Co., Marlborough, MA, USA), SPAXUS ${ }^{\mathrm{TM}}$ stent (Taewoong Medical Co., Gimpo, Korea), and HANARO stent (M.I. Tech, Pyeongtaek, Korea) in earlier implementation. Stent deployment requires the placement of a guidewire (GW) and needle tract dilation using a balloon or a cautery dilator catheter. Electrocautery-enhanced LAMS (EC-LAMS) has been recently developed, which is attached a cautery wire at the tip of the delivery system for the application of cutting current. This system enables puncture, tract dilation, and placement of the stent to be performed using a single device, and has become the most commonly used technique worldwide due to its technical simplicity and safety. Furthermore, advancing the GW through the needle after the puncture sometimes causes the adjacent small intestine to move away from the stomach, resulting in misdeployment. Therefore, more recently, direct insertion of EC-LAMS without a GW has been favored for successful EUS-GE. At present, the only available EC-LAMS is the AXIOS-EC ${ }^{\mathrm{TM}}$ stent (Boston Scientific), which is often referred to as the 'Hot AXIOS.

\section{ENDOSCOPIC ULTRASONOGRAPHY- GUIDED GASTROENTEROSTOMY TECHNIQUES}

The EUS-GE technique was developed through various clinical trials and animal experiments, and at present there are three main techniques: direct EUS-GE, device-assisted EUSGE, and EUS-guided double balloon-occluded gastrojejunostomy bypass (EPASS) (Fig. 1).

\section{Technique 1: Direct EUS-GE (Fig. 1A) , $11-20^{-10}$}

Step 1: EUS is used to carefully identify the duodenum or jejunum adjacent to the gastric body for safe puncturing. However, the intestinal loop is often not clearly visualized by EUS, owing to an insufficiently distended intestine or too much intestinal gas. In such cases, a large amount of liquid (approximately $500 \mathrm{~mL}$ ) should be injected before visualization by EUS. It is desirable to use saline and contrast medium with/without methylene blue rather than water to prevent 


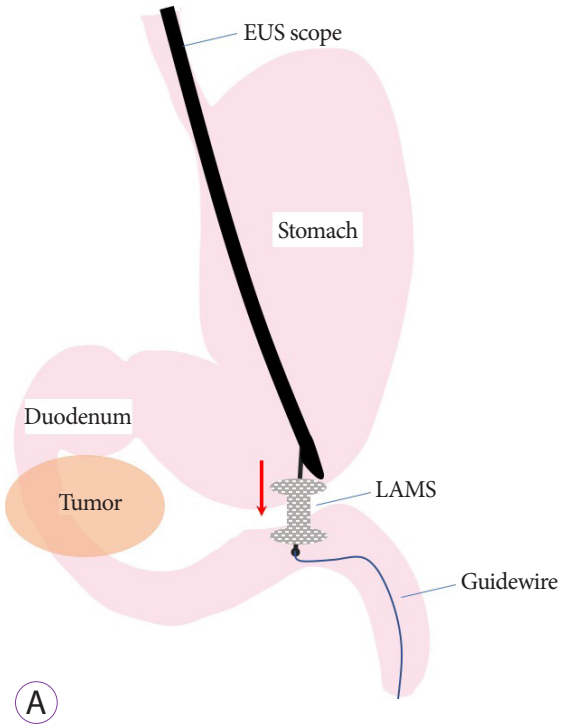

(A)

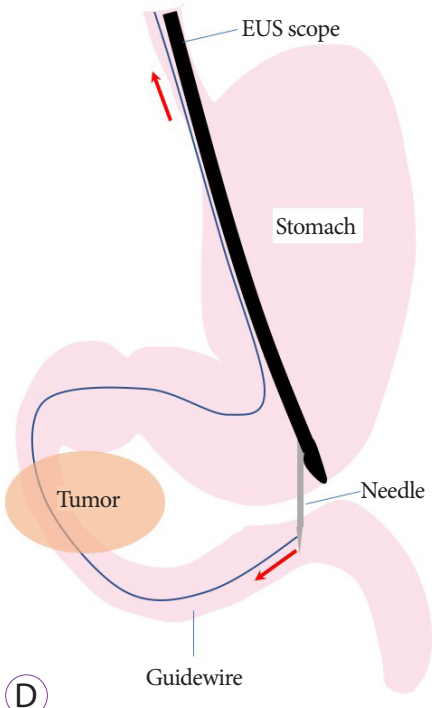

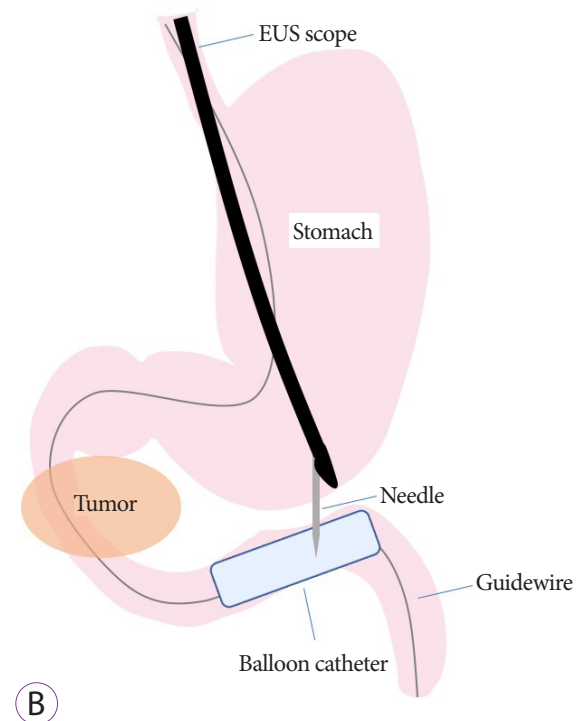

(C)

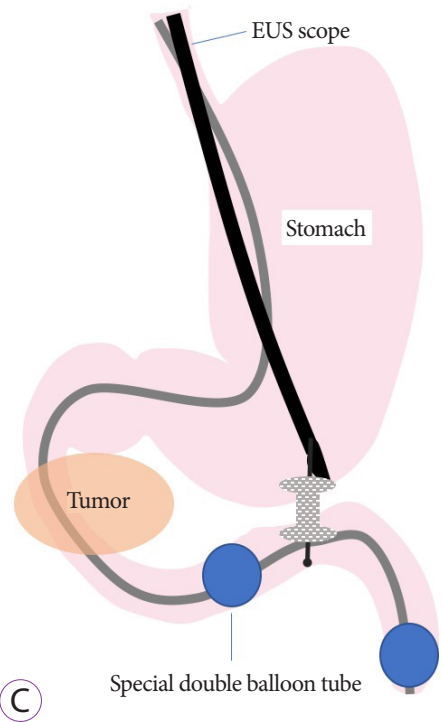

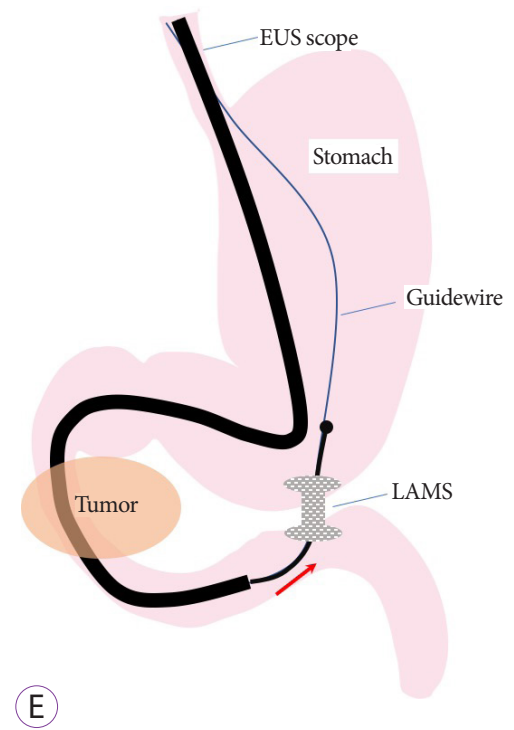

(E)

Fig. 1. Endoscopic ultrasonography (EUS)-guided gastroenterostomy techniques. (A) Direct technique. (B) Balloon-assisted technique. (C) EUS-guided balloonoccluded gastrojejunostomy bypass. (D) Rendezvous guidewire technique. (E) Retrograde deployment technique. LAMS, lumen-apposing self-expandable metal stent.

hyponatremia owing to the absorption of a large amount of water, and to confirm that puncture and stent placement are properly performed at the intended site in a subsequent step. The intestine can be filled with liquid in two ways: injection using a 22-gauge needle as a test puncture just before direct puncture with a 19-guage needle; or use of a preloaded gastrointestinal endoscope and the devices of endoscopic retrograde cholangiopancreatography (ERCP) to fill the intestine from behind the obstruction, or if possible, across the obstruction before insertion of the EUS endoscope.
Step 2: Following confirmation of the distended intestine filled with liquid containing contrast medium under EUS and fluoroscopy, EUS-guided needle puncture using a 19-gauge fine needle is performed. The enterogram, with injection of contrast medium through the needle or aspiration of the blue liquid from the needle, confirms that the target intestine can be correctly punctured to avoid unintentionally puncturing the colon.

Step 3: A 0.025- or 0.035-inch stiff GW is placed downstream of the jejunum through the needle. The needle tract 
is dilated using a balloon or electrocautery dilator following deployment of the LAMS over the GW.

The aforementioned technique is a classical GW-based technique that has been used frequently in previous studies. However, the technique has become simpler owing to the application of EC-LAMS which has enabled one step procedure including puncturing, dilating anastomosis and deployment of the stent, without GW guidance.

\section{Technique 2: Device-assisted EUS-GE (balloon catheter, nasobiliary tube, and ultraslim endoscope) ${ }^{9}$, 11-17}

The device-assisted EUS-GE used a device, such as a balloon catheter, a thin nasobiliary tube, or an ultraslim endoscope, to reliably identify the target small intestine. Among them, the balloon-assisted method, which is often favored and used in previous reports, is described below.

\section{Balloon-assisted technique (Fig. 1B)}

Step 1: A 0.025 - or 0.035 -inch stiff GW is placed downstream of the jejunum beyond the obstruction under gastrointestinal endoscope guidance.

Step 2: After the endoscope is withdrawn, a dilation balloon or a stone extraction balloon catheter is advanced over the wire into the jejunum under fluoroscopic guidance.

Step 3: The inflated balloon is punctured using a 19-gauge fine needle under EUS guidance. Another 0.025- or 0.035-inch stiff GW is placed downstream of the jejunum through the needle.

Step 4: The LAMS is finally deployed over the GW after dilation of the needle tract, as described above.

\section{Technique 3: Endoscopic ultrasonography-guided double balloon-occluded gastrojejunostomy bypass ${ }^{7}$, $10,12,15,16$}

We developed the EPASS technique based on the results of an animal study. ${ }^{23,24}$ Owing to our various experimental results and clinical experience, we currently perform a procedure involving a special double-balloon enteric tube (Create Medic Co., Ltd., Yokohama, Japan). This tube has two balloons which enable wedge the target jejunum, and an independent supply ports, which enables liquid irrigation into the space between the two balloons (Figs. 1C and 2).

Step 1: A standard gastroenteroscope, with an overtube for a single balloon enteroscope (ST-SB1; Olympus Medical Systems, Tokyo, Japan), is advanced behind the stenotic site (pylorus of the stomach or duodenum). The overtube is used to avoid looping of the special double balloon enteric tube in the fornix of the stomach, and facilitate the tube passage through the pyloric-duodenal stenosis. A stiff 0.025 - or 0.035 -inch GW is advanced into the jejunum as far as possible using an ERCP catheter through the working channel of the scope (Fig. 2A).

Step 2: After withdrawal of the endoscope, leaving the overtube and the GW in place, the special balloon tube is perorally inserted over the GW by pulling back the GW, and is placed where the jejunum intended for stent placement is positioned, at the center of the two balloons under fluoroscopic guidance (Fig. 2B). A small amount of contrast medium (approximately $5 \mathrm{~mL}$ for each balloon) followed by saline (approximately $40-50 \mathrm{~mL}$ for each balloon) is injected into the two balloons simultaneously in order to prevent the balloons from moving under fluoroscopy. The injection of saline should be continued until each balloon transforms from a spherical shape into a "barrel shape" (Fig. 2C).

Step 3: After gently removing the overtube from the mouth, the EUS endoscope is advanced into the stomach, and the target jejunum between the two balloons is visualized by EUS after irrigation of tap water or saline $(100-200 \mathrm{~mL})$ with contrast medium (approximately $20 \mathrm{~mL}$ ). The irrigation should be continued until sufficient distension of the target jejunum is observed on the EUS image and fluoroscopy (Fig. 2D).

Step 4: The AXIOS-EC delivery system is directly advanced from the gastric wall into the target jejunum while applying an electrocurrent of cut mode ( $100 \mathrm{~W}, 550 \mathrm{Vp}$ ) provided by the electric generator (ICC200; ERBE, Tübingen, Germany). Finally, the AXIOS-EC is deployed in one step using the technique of intrachannel deployment, which is used for proximal flange deployment of the LAMS in the working channel of the endoscope; this technique may be helpful to prevent misdeployment of the LAMS into the abdominal cavity owing to excessive pulling back of the delivery system during the deployment (Fig. 2E).

\section{Other techniques}

\section{Rendezvous technique}

In the rendezvous technique, instead of passing the GW downstream of the jejunum after the puncture as described above, the GW is trapped in the dilating balloon itself, or in the stone extraction balloon and basket catheter used in the ERCP, and pulled back through the duodenal obstruction and out of the mouth, and thus securing it. ${ }^{25}$ This technique enables the GW to be tensioned by pulling back both ends, and can facilitate smooth insertion of subsequent devices (Fig. 1D).

\section{Retrograde deployment technique}

In the case of pulling the puncturing GW out of the mouth, after withdrawal of the EUS endoscope, a therapeutic endoscope is advanced from the mouth over the GW, traversing 

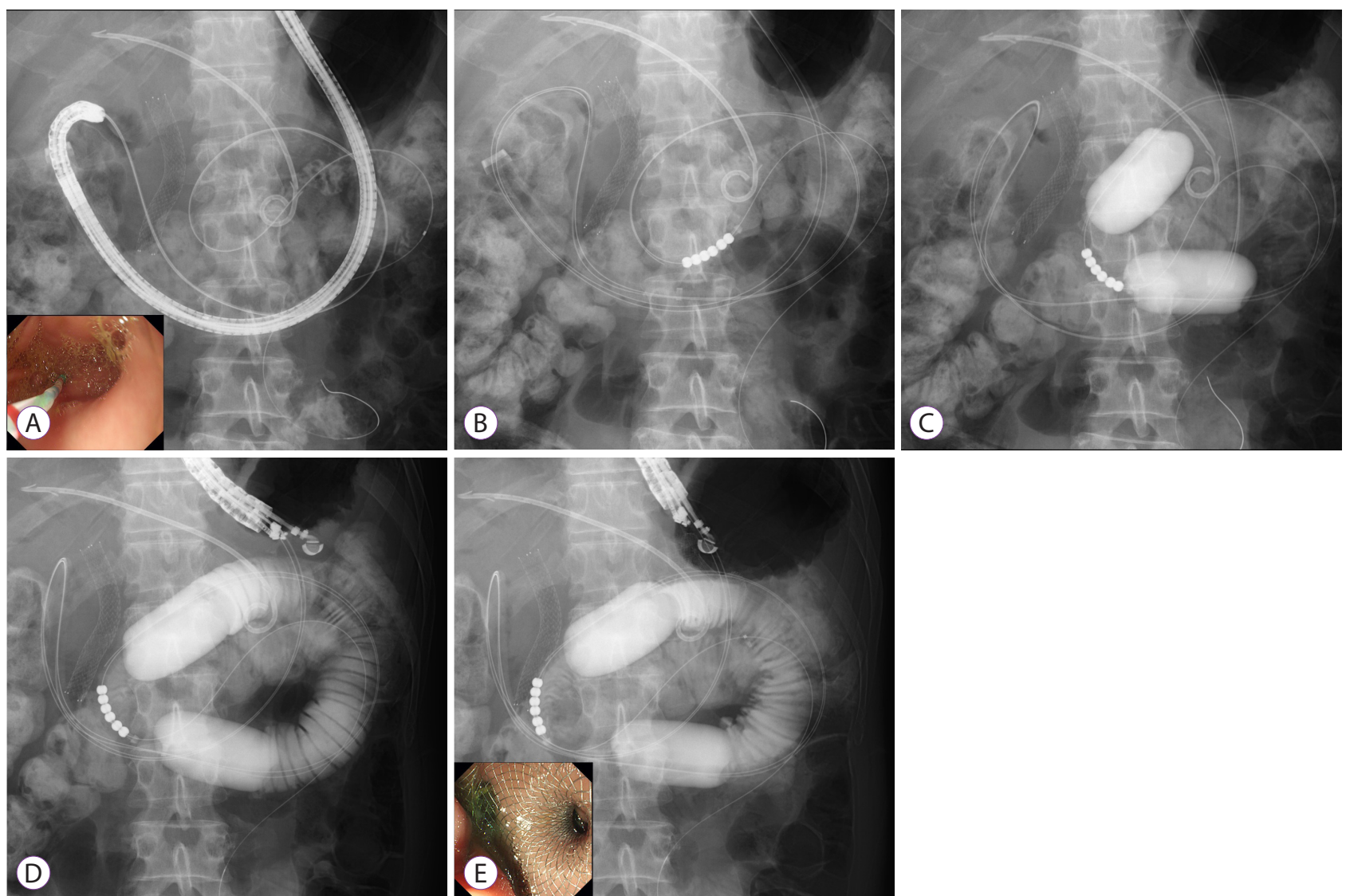

Fig. 2. Endoscopic ultrasonography-guided balloon-occluded gastrojejunostomy bypass. (A) Guidewire advancement across the stricture site into the jejunum. (B) Insertion of the special double-balloon tube. (C) Inflation of the double balloon. (D) Injection of liquids into the target jejunum to induce distension. (E) Deployment of the lumen-apposing self-expandable metal stent.

the obstruction to the punctured site, and then the LAMS is deployed retrogradely from the jejunum. This retrograde placement technique may reduce the risk of misdeployment, because the stomach is less likely to be pushed away, and the gastric gas shadow fluoroscopically enables easier deployment. However, this technique also has disadvantages, in that traversing the endoscope past the obstruction may be difficult in cases of severe duodenal stricture, which may increase the risk of perforation (Fig. 1E). ${ }^{25}$

\section{Outcomes of endoscopic ultrasonography-guided gastroenterostomy}

Previous studies of EUS-GE are summarized in Table 2. The technical and clinical success rates of EUS-GE, regardless of the technique and etiology, have been reported to be $87 \%-$ $100 \%$ and $84 \%-100 \%$, respectively. ${ }^{8-21}$ The rate of AEs was reported to be $0 \%-33 \%$, which includes misdeployment of the stent, bleeding, pneumoperitoneum, peritonitis, abdominal pain, and gastrocolic fistula. Barthet et al. ${ }^{8}$ reported the first case series of successful EUS-GE by using LAMS in three patients; however, they applied pure natural orifice transluminal endoscopic surgery (NOTES), but it was not widely clinically applied due to its complexity and its invasive nature. Khashab et al. first reported a retrospective cohort of EUS-GE with 10 patients, including 3 patients with malignant GOO, treated using the balloon-assisted technique and direct technique. ${ }^{9}$ The technical success rate in this study was $90 \%$, with one unsuccessful procedure, and clinical success in all nine patients in whom the procedure was successful. There were no AEs and no recurrence of symptoms during a mean follow-up of 150 days. Tyberg et al. reported a multicenter international retrospective study of 26 patients, including 17 patients with malignant GOO, in which various EUS-GE techniques, including NOTES, the direct technique, the balloon-assisted technique, and others, were utilized. ${ }^{11}$ Technical success and clinical success were achieved in $92 \%$ and $85 \%$, respectively; in four 
Table 2. Outcomes of Endoscopic Ultrasonography-Guided Gastroenterostomy Using a Lumen-Apposing Self-Expandable Metal Stent

\begin{tabular}{|c|c|c|c|c|c|c|c|c|c|}
\hline Study & Study design & $\begin{array}{l}\text { Sam- } \\
\text { ple } \\
\text { size }\end{array}$ & $\begin{array}{l}\text { Etiology } \\
\text { of GOO }\end{array}$ & Technique & $\begin{array}{l}\text { Tech- } \\
\text { nical } \\
\text { suc- } \\
\text { cess } \\
(\%)\end{array}$ & $\begin{array}{l}\text { Clin- } \\
\text { ical } \\
\text { suc- } \\
\text { cess } \\
(\%)\end{array}$ & $\begin{array}{l}\text { Rate } \\
\text { of } \\
\text { AEs } \\
(\%)\end{array}$ & Details of the AEs & $\begin{array}{l}\text { GOO } \\
\text { recur- } \\
\text { rence/ } \\
\text { reinter- } \\
\text { vention } \\
(\%) \\
\end{array}$ \\
\hline $\begin{array}{l}\text { Barthet et al. } \\
(2015)^{8}\end{array}$ & Case series & 3 & $\begin{array}{c}\text { Benign=1 } \\
\text { Malignant=2 }\end{array}$ & NOTES=3 & 100 & 100 & 33 & Misdeployment=1 & 0 \\
\hline $\begin{array}{l}\text { Khashab et al. } \\
(2015)^{9}\end{array}$ & $\begin{array}{l}\text { Multicenter retrospective } \\
\text { cohort }\end{array}$ & 10 & $\begin{array}{c}\text { Benign }=7 \\
\text { Malignant }=3\end{array}$ & $\begin{array}{c}\text { Direct }=1 \\
\text { Balloon-assisted=9 }\end{array}$ & 90 & 90 & 0 & None & 0 \\
\hline $\begin{array}{l}\text { Itoi et al. } \\
(2016)^{10}\end{array}$ & Prospective cohort & 20 & Malignant $=20$ & EPASS $=20$ & 90 & 90 & 10 & Misdeployment=2 & 0 \\
\hline $\begin{array}{l}\text { Tyberg et al. } \\
(2016)^{11}\end{array}$ & $\begin{array}{l}\text { International multicenter } \\
\text { retrospective cohort }\end{array}$ & 26 & $\begin{array}{c}\text { Benign }=9 \\
\text { Malignant }=17\end{array}$ & $\begin{array}{c}\text { NOTES }=2 \\
\text { Direct=3 } \\
\text { Balloon-assisted=13 } \\
\text { Ultraslim endo- } \\
\text { scope-assisted=5 } \\
\text { Nasobiliary tube-as- } \\
\text { sisted=3 }\end{array}$ & 92 & 85 & 11.5 & $\begin{array}{l}\text { Peritonitis }=1 \\
\text { Bleeding }=1 \\
\text { Pain }=1\end{array}$ & 0 \\
\hline $\begin{array}{l}\text { Khashab et al. } \\
(2017)^{12}\end{array}$ & $\begin{array}{l}\text { International multicenter } \\
\text { retrospective cohort } \\
\text { (EUS-GE vs. surgery) }\end{array}$ & 30 & Malignant $=30$ & $\begin{array}{c}\text { Direct }=2 \\
\text { Balloon-assisted }=6 \\
\text { EPASS }=22\end{array}$ & 87 & 87 & 16 & $\begin{array}{l}\text { Misdeployment }=3 \\
\text { Abdominal pain }=2\end{array}$ & 3 \\
\hline $\begin{array}{l}\text { Perez-Mi- } \\
\text { randa et al. } \\
(2017)^{13}\end{array}$ & $\begin{array}{l}\text { International multicenter } \\
\text { retrospective cohort } \\
\text { (EUS-GE vs. surgery) }\end{array}$ & 25 & $\begin{array}{c}\text { Benign }=8 \\
\text { Malignant }=17\end{array}$ & $\begin{array}{c}\text { Direct=6 } \\
\text { Balloon-assisted=9 } \\
\text { Ultraslim endo- } \\
\text { scope-assisted=7 } \\
\text { Nasobiliary tube-as- } \\
\text { sisted=3 }\end{array}$ & 88 & 84 & 13.6 & $\begin{array}{l}\text { Bleeding }=2 \\
\text { Peritonitis }=1\end{array}$ & 0 \\
\hline $\begin{array}{l}\text { Brewer Guti- } \\
\text { errez et al. } \\
(2017)^{14}\end{array}$ & $\begin{array}{l}\text { Multicenter case series } \\
\text { (EUS-GE with EUS- } \\
\text { BD) }\end{array}$ & 7 & Malignant=7 & $\begin{array}{c}\text { Direct }=5 \\
\text { Balloon-assisted=2 }\end{array}$ & 100 & 100 & 0 & None & 0 \\
\hline $\begin{array}{l}\text { Chen et al. } \\
(2017)^{15}\end{array}$ & $\begin{array}{l}\text { International multicenter } \\
\text { retrospective cohort } \\
\text { (EUS-GE vs. enteral } \\
\text { stenting) }\end{array}$ & 30 & Malignant $=30$ & $\begin{array}{c}\text { Direct }=2 \\
\text { Balloon-assisted }=6 \\
\text { EPASS }=22\end{array}$ & 88 & 84 & 10 & $\begin{array}{l}\text { Bleeding }=2 \\
\text { Peritonitis }=1\end{array}$ & 0 \\
\hline $\begin{array}{l}\text { Chen et al. } \\
(2018)^{16}\end{array}$ & $\begin{array}{l}\text { International multicenter } \\
\text { retrospective cohort (for } \\
\text { only benign diseases) }\end{array}$ & 26 & Benign $=26$ & $\begin{array}{c}\text { Direct }=15 \\
\text { Balloon-assisted }=7 \\
\text { EPASS }=4\end{array}$ & 96 & 84 & 11.5 & $\begin{array}{l}\text { Misdeployment }=2 \\
\text { Gastric leakage }=1\end{array}$ & 4.8 \\
\hline $\begin{array}{l}\text { Chen et al. } \\
(2018)^{17}\end{array}$ & $\begin{array}{l}\text { Multicenter retrospec- } \\
\text { tive cohort (direct vs. } \\
\text { balloon assisted) }\end{array}$ & 74 & $\begin{array}{c}\text { Benign }=25 \\
\text { Malignant }=49\end{array}$ & $\begin{array}{c}\text { Direct }=52 \\
\text { Balloon-assisted }=22\end{array}$ & 94.2 & 92.3 & 6.8 & Misdeployment $=5$ & 9.5 \\
\hline $\begin{array}{l}\text { Ge et al. } \\
(2019)^{18}\end{array}$ & $\begin{array}{l}\text { Retrospective cohort } \\
\text { (EUS-GE vs. enteral } \\
\text { stent) }\end{array}$ & 22 & Malignant $=22$ & Direct $=22$ & 100 & 96 & 18.1 & $\begin{array}{l}\text { Misdeployment=2 } \\
\text { LAMS mesh ero- } \\
\text { sion=1 } \\
\text { Stent ingrowth=1 }\end{array}$ & 4.5 \\
\hline $\begin{array}{l}\text { Kerdsir- } \\
\text { ichairat et al. } \\
(2019)^{19}\end{array}$ & $\begin{array}{l}\text { Multicenter retrospective } \\
\text { cohort }\end{array}$ & 57 & $\begin{array}{c}\text { Benign }=23 \\
\text { Malignant }=34\end{array}$ & Direct $=57$ & 93 & 90 & 3.5 & $\begin{array}{c}\text { Leakage }=1 \\
\text { Hemoperitoneum }=1\end{array}$ & 14.0 \\
\hline $\begin{array}{l}\text { James et al. } \\
(2020)^{20}\end{array}$ & $\begin{array}{l}\text { Retrospective cohort (for } \\
\text { only benign diseases) }\end{array}$ & 22 & Benign $=22$ & Direct $=14$ & 95.4 & 95.4 & 18.2 & $\begin{array}{l}\text { Abdominal pain }=1 \\
\text { Severe bleeding }=1 \\
\text { Stent migration }=1 \\
\text { Penetrating colon }=1\end{array}$ & 22.7 \\
\hline $\begin{array}{l}\text { Wannhoff et } \\
\text { al. }(2020)^{21}\end{array}$ & Retrospective cohort & 35 & $\begin{array}{c}\text { Benign }=2 \\
\text { Malignant }=33\end{array}$ & $\begin{array}{l}\text { Direct }=36 \\
\text { Others }=2\end{array}$ & 80 & 74.3 & 14.3 & $\begin{array}{c}\text { Peritonitis }=3 \\
\text { Perforation=1 } \\
\text { Misdeployment=1 }\end{array}$ & 10 \\
\hline
\end{tabular}

AEs, adverse events; EPASS, endoscopic ultrasonography-guided double balloon-occluded gastrojejunostomy bypass; EUS-BD, endoscopic ultrasonography-guided biliary drainage; EUS-GE, endoscopic ultrasonography-guided gastroenterostomy; GOO, gastric outlet obstruction; LAMS, lumen-apposing self-expandable metal stent; NOTES, natural orifice transluminal endoscopic surgery. 
patients in whom clinical success was not achieved, two had persistent nausea and vomiting despite a patent EUS-GE, and required enteral feeding for nutrition, one died before the initiation of an oral diet, and one underwent surgery for suspected perforation. AEs occurred in three patients (11.5\%), and after a mean follow-up of 7.9 weeks, no symptom recurrence was noted in any of the clinically successful cases. Itoi et al. ${ }^{10}$ reported a prospective cohort study in which the EPASS technique was performed in 20 cancer patients. The overall technical success and clinical success rates were both $90 \%$, whereas the one-step procedure had a higher technical success rate than the two-step procedure (100\% vs. $81.8 \%$ ). In addition, no stent occlusion or migration occurred in any of the patients in whom successful stent deployment was performed during a median follow-up of 100 days. Furthermore, Wannhoff et al. ${ }^{21}$ reported a retrospective analysis of factors associated with successful procedures in 35 patients in whom the direct technique was performed. In 10 patients, the LAMS was inserted over the GW, in 22 patients, direct puncture using the electrocautery delivery system of the LAMS was performed, and other techniques were used in 2 patients. The technical success rate, clinical success rate, and AEs were $80.0 \%, 74.3 \%$, and $14.3 \%$, respectively. As the number of cases increased, the technical success rate improved, and the procedure time was reduced. Regarding factors associated with technical success, the distance between the two lumina connected with the LAMS was significantly shorter in patients with successful surgery than in those without ( $9 \mathrm{~mm}$ vs. $20 \mathrm{~mm}, p=0.004$ ); this distance was only identified as a predictor of success on multivariate analysis. Regarding comparison with the EUS-GE technique, Chen et al. ${ }^{17}$ compared the efficacy and safety of the direct technique with the balloon-assisted technique. There were no significant differences between the two groups with regards to the technical success, clinical success, rate of AEs, need for repeated intervention, or survival, and only the mean procedure time was significantly lower in the direct technique group than in the balloon-assisted technique group (35.7 $\mathrm{min}$ vs. $89.9 \mathrm{~min}$ ). In terms of the long-term results of EUS-GE, Kerdsirichairat et al. ${ }^{19}$ reported a retrospective cohort of 57 patients who underwent the direct technique, with a median follow-up of 196 days in the malignant GOO group (34 patients) and 319.5 days in the benign GOO group (23 patients). Technical success and clinical success were achieved in $93 \%$ and $89.5 \%$, respectively. There were $3.5 \%$ ( 2 cases) of AEs, including 1 stent leakage (severe) and 1 hemoperitoneum (moderate), and 8 of the 53 patients (15.1\%) required an unplanned reintervention, of whom, 2 had stent occlusion and 6 had patent stents at repeat upper endoscopy during the follow-up period.

\section{Comparison with traditional palliative therapies}

Two previous studies have compared EUS-GE and SGJ. Khashab et al. ${ }^{12}$ reported a multicenter international retrospective comparative study of EUS-GE $(n=30)$ and open gastrojejunostomy $(n=63)$ in patients with malignant GOO. Although there was a difference in the patients' backgrounds, in that the EUS-GE group had more patients with peritoneal carcinomatosis than the surgery group, the technical success rate was lower in the EUS-GE group ( $87 \%$ vs. $100 \%$ ), but the clinical success rate was not significantly different between the two groups ( $87 \%$ vs. $90 \%$ ). Moreover, the rate of AEs was also lower in the EUS-GE group than in the surgery group ( $16 \%$ vs. $25 \%$ ). In terms of the rate of recurrent GOO (3\% vs. $14 \%$ ) and the mean time to reintervention (88 days vs. 121 days), there were no significant differences between the two groups. Perez-Miranda et al. ${ }^{13}$ reported a multicenter international retrospective comparative study of EUS-GE $(n=25)$ and laparoscopic gastrojejunostomy $(n=29)$ in patients with malignant and benign GOO. They reported no significant difference in the technical success rate between the two groups (EUS-GE: $88 \%$ vs. laparoscopic gastrojejunostomy: $100 \%$, $p=0.11$ ), but the rate of AEs was significantly lower in the EUS-GE group ( $12 \%$ vs. $41 \%, p=0.0386$ ). Furthermore, in terms of the expected cost, EUS-GE was significantly cheaper than laparoscopic gastrojejunostomy ( $\$ 4,905.5$ vs. $\$ 14,778.80$, $p<0.00001$ ).

Two recent studies have compared EUS-GE with EES. Chen et al. ${ }^{15}$ reported a multicenter international retrospective comparative study between EUS-GE $(n=30)$ and EES $(n=52)$ in patients with malignant GOO. There was no difference in the technical success rate (EUS-GE: $86.7 \%$ vs. EES: $94.2 \%$, $p=0.2)$, clinical success rate ( $83.3 \%$ vs. $67.3 \%, p=0.12)$, rate of AEs ( $16.7 \%$ vs. $11.5 \%, p=0.5)$, and severe AEs ( $10 \%$ vs. $9.6 \%)$ between the two groups. However, the recurrence of GOO symptoms and the need for reintervention were significantly lower in the EUS-GE group than in the ES group (4.0\% vs. $28.6 \%, p=0.015$ ), even in cases where the endoscopically placed stent was larger $(15 \mathrm{~mm}$ in diameter in EUS-GE vs. 22 or $20 \mathrm{~mm}$ in EES). Furthermore, multivariate analysis demonstrated that EES was independently associated with the need for reintervention (odds ratio, $12.8 ; p=0.027$ ). Ge et al. ${ }^{18}$ performed a retrospective comparative study between EUSGE and EES in 100 consecutive patients, including 22 patients with EUS-GE and 78 patients with EES. The primary outcome of this study was the rate of reintervention for recurrent GOO symptoms, which was higher in the EES group than in the EUS-GE group (EUS-GE: $8.3 \%$ vs. EES: $32.0 \%, p=0.021$ ). Technical success was achieved in $100 \%$ of the patients in both groups; however, the initial clinical success rate was higher in 
the EUS-GE group than in the EES group ( $95.8 \%$ vs. $76.3 \%$, $p=0.042$ ). The authors concluded that EUS-GE may be suitable for selected patients with malignant GOO in centers with extensive experience.

\section{Future perspectives}

The limited available evidence, as mentioned above, indicate that EUS-GE is an effective and safe technique, and has the potential to become an alternative to SGJ and EES in patients with malignant GOO. Previously, the prognosis of patients with GOO owing to unresectable pancreatic cancer was 12 weeks ${ }^{2}$; however, but with the recent development of chemotherapy, a long-term prognosis of nearly 1 year can now be expected, and therefore, a minimally invasive and long-term patency procedure to relieve GOO is required. More recently, EUS-GE has been attempted in patients with benign diseases, and patency of the LAMS for more than 1 year in some patients has been reported, demonstrating the durability of EUSGE. ${ }^{16,20}$ In the near future, even for patients with malignant diseases, long-term stent patency of EUS-GE is expected to maintain a patient's nutritional condition, and longer survival times are expected to be achieved with continuous and effective chemotherapy.

Identification of the targeted intestinal loop is crucial for successful deployment, and although various techniques for EUS-GE have been developed and improved, the optimal technique remains unclear. Theoretically, it is impossible to perform a secure puncture into the collapsed duodenum or jejunum without some preparation to distend the small bowel, such as by using the water-filling luminal technique. However, even if a large amount of water is injected into the small bowel, the position of the small bowel moves and gradually collapses owing to the flow of water to the colon by peristalsis, even when antimotility drugs are administered. Therefore, if possible, the use of a double-balloon enteric tube is preferable to maintain distension of the intestine owing to additional injection of fluid, when the small bowel collapses after needle puncture or tract dilation. However, complete GOO may preclude advancement of the GW and over-the-wire devices into the distal jejunum. In cases of complete GOO, predilation using a dilating balloon catheter before inserting over-the-wire devices appears to be useful.

Some previous reports have reported other applications of EUS-GE. The causes of malignant GOO include periampullary malignancy, which often results in not only duodenal obstruction but also biliary stricture, and the management of both is necessary for long-term survival and maintenance of a patient's QOL. At present, patients with double obstruction of the duodenum and the biliary duct are often treated using endoscopic double stenting, which involves endoscopic biliary stent placement and EES, or double bypass surgery. ${ }^{26}$ In particular, in endoscopic double stenting, it is difficult to manage both the biliary drainage and duodenal obstruction, because the tumor invasion disturbs successful stent placement and stent patency. Brewer Gutierrez et al. ${ }^{14}$ reported a case series of seven patients with obstruction of the duodenum and biliary duct, in which double endoscopic bypass with EUS-GE and EUS-guided biliary drainage (EUS-BD) was performed. The advantage of this novel method is that the two bypasses are not across the tumor, and hence there is a possibility that long-term patency can be obtained without stent occlusion due to tumor invasion. Furthermore, the QOL of the patient is increased if the procedure can be performed in a single session. In this report, four patients underwent EUS-guided gallbladder drainage and two patients underwent EUS-guided choledochoduodenostomy (EUS-CDS) with the EUS-BD technique. Considering the physiological flow, EUS-guided hepaticogastrostomy may be more suitable than EUS-CDS in a patient with GOO. Further trials involving a larger number of patients, as well as a comparison with double stenting, are required in the future.

The usefulness of EUS-GE has also been reported for afferent loop syndrome. Brewer Gutierrez et al. ${ }^{14}$ described a multicenter study of EUS-GE for 18 patients with afferent loop syndrome. Resolution of symptoms was observed in $88.9 \%$ of patients, and improvement to enable hospital discharge was found in $11.1 \%$ of the patients. Technical success was achieved in $100 \%$ of the patients, with a mean procedure time of 29.7 minutes. Compared to enteroscopy-assisted stent placement, patients treated with EUS-GE required fewer reinterventions ( $16.6 \%$ vs. $76.5 \%, p<0.001)$.

A new technique has been proposed for challenging cases of EUS-GE, since EUS-GE for GOO is sometimes difficult, particularly in situations with an intervening colon, a long distance between the distant small bowel and the gastric wall, and adhesions trapping the small bowel in the right side of the abdomen. Furthermore, EES is also limited in cases where the stricture is very long or sharply angulated; however, Irani et al. reported successful EUS-guided duodenojejunostomy or jejunojejunostomy in such patients. ${ }^{27}$ Moreover, the endoscopic position often becomes long, which makes deployment of the LAMS challenging. The authors proposed tips to overcome such situations, by withdrawal of the LAMS to a straight endoscopic position, advancing the LAMS out of the channel, and then repositioning it for puncture. 


\section{CONCLUSIONS}

In the present review, we have discussed the feasibility and future perspectives of EUS-GE using LAMS. This procedure has not yet been optimized, and there are a few limitations that need to be overcome. All reports of EUS-GE have been published only by experts of the procedure because the currently followed procedure is technically difficult and must be improved and simplified further to facilitate its use in clinical practice. LAMS design must also be improved because the currently available LAMSs have a maximum diameter that does not appear to be appropriate for EUS-GE, which usually requires a bigger anastomosis and minimal risk of stent obstruction and migration. Furthermore, clinical prospective randomized-control trials comparing EUS-GE and EES or SGJ are warranted. In the near future, EUS-GE is expected to become the gold standard treatment for malignant GOO, owing to its lower invasiveness than SGJ, and longer durability than EES.

\section{Conflicts of Interest}

Takao Itoi is a consultant for Boston Scientific. The other authors have no financial conflicts of interest.

ORCID

Takayoshi Tsuchiya: https://orcid.org/0000-0002-2586-0635

Shuntaro Mukai: https://orcid.org/0000-0001-5737-620X

Yuichi Nagakawa: https://orcid.org/0000-0003-1169-8160

Takao Itoi: https://orcid.org/0000-0002-9433-8437

\section{REFERENCES}

1. Tringali A, Didden P, Repici A, et al. Endoscopic treatment of malignant gastric and duodenal strictures: a prospective, multicenter study. Gastrointest Endosc 2014;79:66-75.

2. Conio M, Demarquay JF, De Luca L, Marchi S, Dumas R. Endoscopic treatment of pancreatico-biliary malignancies. Crit Rev Oncol Hematol 2001;37:127-135.

3. Tendler DA. Malignant gastric outlet obstruction: bridging another divide. Am J Gastroenterol 2002;97:4-6.

4. Maetani I, Inoue H, Sato M, Ohashi S, Igarashi Y, Sakai Y. Peroral insertion techniques of self-expanding metal stents for malignant gastric outlet and duodenal stenoses. Gastrointest Endosc 1996;44:468-471.

5. Khashab M, Alawad AS, Shin EJ, et al. Enteral stenting versus gastrojejunostomy for palliation of malignant gastric outlet obstruction. Surg Endosc 2013;27:2068-2075.

6. Jeurnink SM, Steyerberg EW, van Hooft JE, et al. Surgical gastrojejunostomy or endoscopic stent placement for the palliation of malignant gastric outlet obstruction (SUSTENT study): a multicenter randomized trial. Gastrointest Endosc 2010;71:490-499.

7. Itoi $\mathrm{T}, \mathrm{Baron} \mathrm{TH}$, Khashab MA, et al. Technical review of endoscopic ultrasonography-guided gastroenterostomy in 2017. Dig Endosc 2017;29:495-502.

8. Barthet M, Binmoeller KF, Vanbiervliet G, Gonzalez JM, Baron TH, Berdah S. Natural orifice transluminal endoscopic surgery gastroen- terostomy with a biflanged lumen-apposing stent: first clinical experience (with videos). Gastrointest Endosc 2015;81:215-218.

9. Khashab MA, Kumbhari V, Grimm IS, et al. EUS-guided gastroenterostomy: the first U.S. clinical experience (with video). Gastrointest Endosc 2015;82:932-938.

10. Itoi T, Ishii K, Ikeuchi N, et al. Prospective evaluation of endoscopic ultrasonography-guided double-balloon-occluded gastrojejunostomy bypass (EPASS) for malignant gastric outlet obstruction. Gut 2016;65:193195.

11. Tyberg A, Perez-Miranda M, Sanchez-Ocaña R, et al. Endoscopic ultrasound-guided gastrojejunostomy with a lumen-apposing metal stent: a multicenter, international experience. Endosc Int Open 2016;4:E276-E281.

12. Khashab MA, Bukhari M, Baron TH, et al. International multicenter comparative trial of endoscopic ultrasonography-guided gastroenterostomy versus surgical gastrojejunostomy for the treatment of malignant gastric outlet obstruction. Endosc Int Open 2017;5:E275-E281.

13. Perez-Miranda M, Tyberg A, Poletto D, et al. EUS-guided gastrojejunostomy versus laparoscopic gastrojejunostomy: an international collaborative study. J Clin Gastroenterol 2017;51:896-899.

14. Brewer Gutierrez OI, Nieto J, Irani S, et al. Double endoscopic bypass for gastric outlet obstruction and biliary obstruction. Endosc Int Open 2017;5:E893-E899.

15. Chen YI, Itoi T, Baron TH, et al. EUS-guided gastroenterostomy is comparable to enteral stenting with fewer re-interventions in malignant gastric outlet obstruction. Surg Endosc 2017;31:2946-2952.

16. Chen YI, James TW, Agarwal A, et al. EUS-guided gastroenterostomy in management of benign gastric outlet obstruction. Endosc Int Open 2018;6:E363-E368.

17. Chen YI, Kunda R, Storm AC, et al. EUS-guided gastroenterostomy: a multicenter study comparing the direct and balloon-assisted techniques. Gastrointest Endosc 2018;87:1215-1221

18. Ge PS, Young JY, Dong W, Thompson CC. EUS-guided gastroenterostomy versus enteral stent placement for palliation of malignant gastric outlet obstruction. Surg Endosc 2019;33:3404-3411.

19. Kerdsirichairat T, Irani S, Yang J, et al. Durability and long-term outcomes of direct EUS-guided gastroenterostomy using lumen-apposing metal stents for gastric outlet obstruction. Endosc Int Open 2019;7:E144-E150.

20. James TW, Greenberg S, Grimm IS, Baron TH. EUS-guided gastroenteric anastomosis as a bridge to definitive treatment in benign gastric outlet obstruction. Gastrointest Endosc 2020;91:537-542.

21. Wannhoff A, Ruh N, Meier B, Riecken B, Caca K. Endoscopic gastrointestinal anastomoses with lumen-apposing metal stents: predictors of technical success. Surg Endosc 2020 May 1 [Epub]. https://doi. org/10.1007/s00464-020-07594-5.

22. Binmoeller KF, Shah JN. Endoscopic ultrasound-guided gastroenterostomy using novel tools designed for transluminal therapy: a porcine study. Endoscopy 2012;44:499-503.

23. Itoi T, Itokawa $\mathrm{F}$, Uraoka $\mathrm{T}$, et al. Novel EUS-guided gastrojejunostomy technique using a new double-balloon enteric tube and lumen-apposing metal stent (with videos). Gastrointest Endosc 2013;78:934-939.

24. Itoi T, Ishii K, Tanaka R, Umeda J, Tonozuka R. Current status and perspective of endoscopic ultrasonography-guided gastrojejunostomy: endoscopic ultrasonography-guided double-balloon-occluded gastrojejunostomy (with videos). J Hepatobiliary Pancreat Sci 2015;22:3-11.

25. Irani S, Itoi T, Baron TH, Khashab M. EUS-guided gastroenterostomy: techniques from East to West. VideoGIE 2020;5:48-50.

26. Tonozuka R, Itoi T, Sofuni A, Itokawa F, Moriyasu F. Endoscopic double stenting for the treatment of malignant biliary and duodenal obstruction due to pancreatic cancer. Dig Endosc 2013;25(Suppl 2):100-108.

27. Irani S, Khashab M. Gastric outlet obstruction: when you cannot do an endoscopic gastroenterostomy or enteral stent, try an endoscopic duodenojejunostomy or jejunojejunostomy. VideoGIE 2020;5:125-128. 